\title{
Combination of three typing methods for the molecular epidemiology of Aspergillus fumigatus infections
}

\author{
E. RODRIGUEZ, F. SYMOENS, $\ddagger$ P. MONDON, ${ }^{\dagger}$ M. MALLIE, M-A. PIENS, ${ }^{*}$ B. LEBEAU, $\dagger$ A-M. \\ TORTORANO,§ F. CHAIB, * A. CARLOTTI,* J.VILLARD,* M-A. VIVIANI,§ F. CHAPUIS, * N. \\ NOLARD, $\ddagger$ R. GRILLOT† and J-M. BASTIDE \\ (European Research Group on Biotype and Genotype of Aspergillus)
}

\begin{abstract}
Laboratoire d'Immunologie et Parasitologie, Faculté de Pharmacie, 15, Avenue Charles Flahault, 34060 Montpellier Cedex 2, France, * Laboratoire de Mycologie Fondamentale et Appliquée aux Biotechnologies Industrielles, Faculté de Pharmacie, 8 Avenue Rockefeller, 69373 Lyon Cedex 08, France, †ParasitologieMycologie Médicale et Moléculaire, Laboratoire Relations Hôte-Agents Pathogènes (UPRES-A CNRS 5082), Université Joseph Fourier, Faculté de Médecine, Domaine de la Merci, 38706 La Tronche, France, ¥Institut Scientifique de la Santé Louis Pasteur, Section Mycology, 14 Rue Juliette Wytsman, B-1050 Bruxelles, Belgium and §Instituto de Igiene e Medicina Preventiva, via F. Sforza 35, 20122 Milan, Italy
\end{abstract}

This study investigated the source of infection and strain relatedness of Aspergillus fumigatus isolates from bronchial colonisation and invasive aspergillosis (IA) in four transplant patients. Environmental isolates from the patient's home and from the hospital and infecting isolates were obtained for patient $A$ who developed IA. Clinic environmental and colonising isolates were obtained for patient B. Sequential isolates were obtained from various organs from patient $C$ who developed IA and also from patient $D$ who had a bronchitic aspergillosis that developed into IA. Ninety-one $A$. fumigatus isolates were analysed by three typing methods: multi-locus enzyme electrophoresis (MLEE), random amplified polymorphic DNA (RAPD) and sequencespecific DNA primers (SSDP). The three combined typing methods demonstrated a greater differentiation of isolates than the typing methods used separately or in pairs. This demonstrated the genotypic variability of $A$. fumigatus and facilitated better epidemiological analysis. Large polymorphisms were demonstrated for each patient isolate between and colonies within various samples. The relatedness of the isolates suggested nosocomially acquired aspergillosis for patient $B$, but the source of infection for patient $A$ remained unclear. The results suggested at least three multiple infections among the four patients. This study enabled the identification of the source of infection and strain relatedness, which in turn facilitates the development of preventive measures for patient management in the future.

\section{Introduction}

Aspergillus fumigatus is a significant nosocomial pathogen in immunocompromised patients, associated with a high mortality rate $(85 \%)$ even when appropriate treatment is used [1,2]. The incidence of aspergillosis has increased significantly over the past two decades in

Received 15 Jan. 1998; revised version accepted 21 June 1998.

Corresponding author: Professor R. Grillot, Department de Parasitologie-Mycologie Médicale et Moléculaire, Laboratoire Relations Hôte-Agents Pathogènes (UPRES-A CNRS 5082), Universite Joseph Fourier, Faculté de Médecine, Domaine de la Merci, 38706 La Tronche, France. parallel with the number of immunocompromised patients [3-6]. This has been combined with an overall increase in spore counts during periods of building work, both inside and outside hospitals, often resulting in clusters of infections [7]. However, multiple $A$. fumigatus genotypes have been demonstrated to be predominant and persistent over long periods in different geographical centres [7-9]. Because $A$. fumigatus is not a usual human pathogen or commensal, the origin of infection must be exogenous. Therefore, the analysis of multiple isolates from infected patients and the environment is needed to determine the local source of infection, to identify recurrent infections and thus facilitate prevention. Although, as yet, an ideal epidemiological typing 
technique applicable to a wide range of $A$. fumigatus isolates is not available, several molecular typing methods have been examined previously. These include DNA fingerprinting techniques such as arbitrary fragment length polymorphism analysis [10-20], restriction fragment length polymorphism analysis (RFLP) $[8,15,21]$ and moderately repetitive sequences analysis (MRS) [22]. Protein electrophoretic typing methods, such as immunoblotting $[23,24]$ or multi-locus enzyme electrophoresis (MLEE) $[9,15,20,25,26]$ have proved to be useful. However, care has to be taken when studying unrelated isolates by a single method. The combination of typing methods appears to provide better discriminatory power $[10,15,20,27-29]$. Therefore, this study applied and then combined MLEE, random amplified polymorphic DNA (RAPD) and sequence-specific DNA primers (SSDP), which is a new method derived from the second [16-18] to type several isolates from four immunocompromised patients infected by $A$. fumigatus. The study attempted to determine the polymorphism of infective strains and to identify the precise origin of infective strains isolated from two patients. This also will provide new insights into A. fumigatus ecology.

\section{Materials and methods}

\section{Patients and A. fumigatus isolates}

Patients A and B were monitored in France (Hospital A. Michallon, Grenoble). Patient A had invasive aspergillosis (IA) after a bone marrow transplant. Patient B had bronchopulmonary colonisation after a lung transplant. Patient $\mathrm{C}$ was monitored in Italy (Ospedale Majore, Milan). He had IA after a liver transplant. Patient D was monitored in France (Hospice Civil de Lyon, Lyon) and had IA following bronchopulmonary colonisation after a lung transplant. A. fumigatus isolates were obtained from clinical samples examined at the three European hospital centres (Grenoble, Milan, Lyon) and environmental samples related to patients A and B were also collected. All isolates were identified in these centres by conventional techniques based on classical morphological criteria [30] and then lyophilised. All A. fumigatus isolates and related patients medical records are kept by the Institute of Hygiene and Epidemiology, section of Mycology (IHEM culture collection, Brussels, Belgium).

\section{MLEE}

The $A$. fumigatus isolates were cultivated at $42^{\circ} \mathrm{C}$ on minimal Sabouraud solid medium - (w/v: glucose $0.2 \%$, Bactopeptone (Gibco) $0.1 \%, \mathrm{KH}_{2} \mathrm{PO}_{4} \quad 0.1 \%$, $\mathrm{MgSO}_{4}$ 0.1\%, Select Agar (Gibco) 2\%). Cultures were incubated on a gyratory shaker, $150 \mathrm{rpm}$, at $32^{\circ} \mathrm{C}$ in $500-\mathrm{ml}$ flasks containing $150 \mathrm{ml}$ of minimal Sabouraud liquid medium (Diagnostics Pasteur), $\mathrm{pH}$ 8.0, sterilised by autoclaving. After incubation for 4 days, the mycelial growth was harvested in phosphate buffered saline (PBS; $0.05 \mathrm{M}$ sodium phosphate, $\mathrm{pH} 7.4$, containing
$\mathrm{NaCl} 1 \% \mathrm{w} / \mathrm{v}$ ). Then, the mycelium was washed twice with sterile distilled water. The mycelial fragments were mechanically disrupted for $2 \mathrm{~min}$ with glass beads $(0.25 \mathrm{~mm}$ diameter $)$ in a B. Braun ScienceTec MSK homogeniser (San Francisco, CA, USA). Cellular debris was removed by centrifugation at $12000 \mathrm{~g}$ for $30 \mathrm{~min}$ at $0^{\circ} \mathrm{C}$. The supernate of each strain was lyophilised, suspended in a minimal volume of distilled water, and stored in small volumes at $-20^{\circ} \mathrm{C}$ until used in the MLEE. To avoid protease activities and then to ensure enzyme stability the protein extraction and electrophoresis steps were done at $4^{\circ} \mathrm{C}$. Starch gel electrophoresis and specific enzyme staining were performed according to published protocols [31-33]. In this study, 11 enzymic systems were analysed: peptidase A (PEP.A, E.C. 3.4.11; substrate: VAL-LEU), peptidase B (PEP.B, E.C. 3.4.11; substrate: LEU-GLY-GLY), glucose phosphate isomerase (GPI, E.C. 5.3.1.9), phosphoglucomutase (PGM, E.C. 2.7. 5.1), hexokinase (HK, E.C. 2.7.1.1), isocitrate dehydrogenase (IDH, E.C. 1.1.1.42), malic enzyme (ME, E.C. 1.1.1.40), aspartate amino transferase (AAT, E.C. 2.6.1.1), glutamate dehydrogenase (GDH, E.C. 1.4.1.2), purine-nucleoside phosphorylase (NP, E.C. 2.4.2.1) and pyruvate kinase (PK, E.C. 2.7.1.40). Alleles were represented in increasing order of anodal mobility. Each isolate was characterised by its allelic combination at the different polymorphic enzymic loci encountered (Fig. 1). Distinctive multi-locus variants were designated as MLEE types.

\section{SSDP}

Amplification was performed with purified DNA as described previously $[21,22,24]$. The reaction mixture $(30 \mu \mathrm{l})$ contained $10 \mathrm{mM}$ Tris- $\mathrm{HCl}(\mathrm{pH} 8.3), 50 \mathrm{mM}$ $\mathrm{KCl}, 1.5 \mathrm{mM} \mathrm{MgCl}$, Tween $200.005 \%$, NP-40 $0.005 \%, 75 \mu \mathrm{M}$ of each dNTP (Boehringer), $0.5 \mathrm{U}$ of Replitherm $^{\mathrm{R}}$ DNA polymerase (Epicentre), $50 \mathrm{ng}$ of DNA template and $0.2 \mu \mathrm{M}$ of random primer ( 10 mers) or $0.1 \mu \mathrm{M}$ of each specifically designed primer (20 mer). Each reaction mixture was overlaid with mineral oil $(30 \mu \mathrm{l})$ to prevent evaporation. Amplifications were performed in a DNA thermal cycler (Perkin Elmer-480) after an initial heat denaturation $\left(95^{\circ} \mathrm{C}\right.$ for $6 \mathrm{~min}$ ). For arbitrary short primers, 40 cycles consisting of $0.3 \mathrm{~min}$ at $95^{\circ} \mathrm{C}, 0.5 \mathrm{~min}$ at $36^{\circ} \mathrm{C}$ and $1 \mathrm{~min}$ at $63^{\circ} \mathrm{C}$ were used, while for specific long primers, 30 cycles consisting of $0.3 \mathrm{~min}$ at $95^{\circ} \mathrm{C}, 0.5 \mathrm{~min}$ at $55^{\circ} \mathrm{C}$ and $1 \mathrm{~min}$ at $72^{\circ} \mathrm{C}$ were performed. Amplification products were resolved by electrophoresis on agarose $1.5 \%$ gels stained with ethidium bromide $0.5 \mathrm{mg} / \mathrm{ml}$. The DNA template amplified by RAPD with a short primer (10 mers) and $2 \mu \mathrm{Ci}\left[\alpha^{35} \mathrm{~S}\right] \mathrm{dATP}$ (Amersham) was separated by electrophoresis on an acrylamide $(6 \%)$ urea $(50 \%)$ sequencing gel and subjected to autoradiography. From the RAPD patterns, bands of interest were collected, the gel was homogenised with a conical grinder and $10 \mu \mathrm{l}$ of the mixture were amplified directly as described previously, without further DNA amplification [18]. The amplification products were 


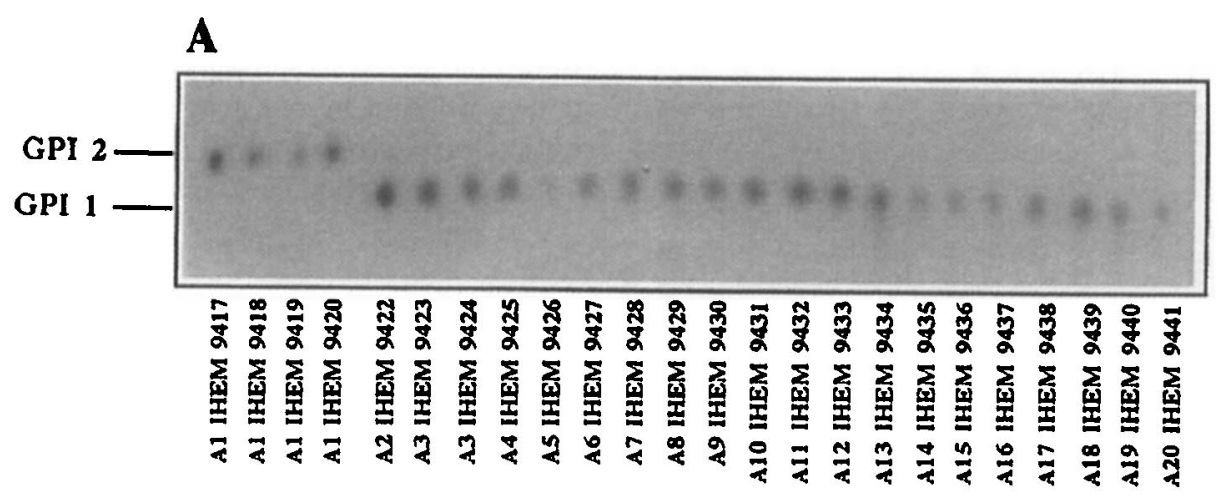

B

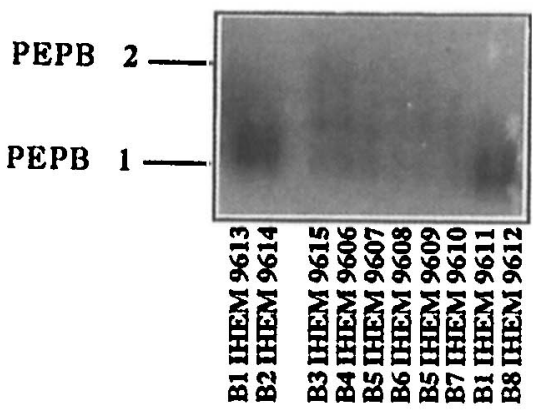

$\mathbf{C}$

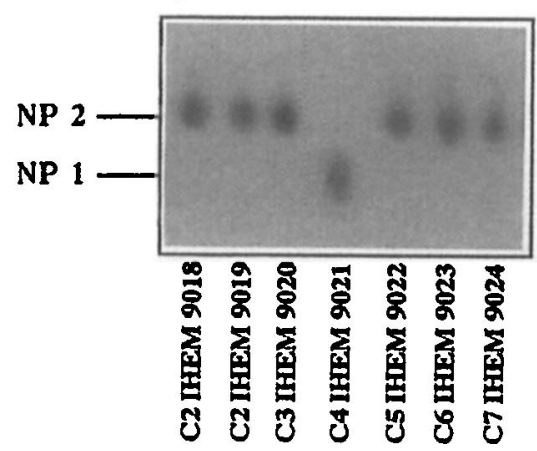

Fig. 1. Alleles are represented by their anodal migration. (A) polymorphism observed in A. fumigatus for patient A with GPI as the enzymic system. Allele GPI 2 completely differentiates the strain infecting patient A with respect to environmental isolates. (B) Polymorphism observed in $A$. fumigatus for patient B with PEP B as the enzymic system. (C) Polymorphism observed in A. fumigatus for patient C with NP as the enzymic system.

cloned with a TA cloning kit (Invitrogen). The insert DNA was sequenced with a Sequenase 2.0 kit (United States Biochemicals) and $\left[\alpha^{35} \mathrm{~S}\right]$ dATP (Amersham). On the basis of the sequence, six oligonucleotide primer sequences were selected including the sequence of the RAPD primers (10 mers) plus 10 bases characteristic of the fragment excised and synthesised by Eurogentec (Belgium). The sequence-specific DNA primers (SSDP) were then used in higher stringency PCR (annealing temperature $55^{\circ} \mathrm{C}$ ) to type the isolates according to the presence or absence of one band visualised on an agarose $1.5 \%$ gel. Distinctive profiles were designated as SSDP types (Fig. 2). The sequences of the primers used are listed in Table 3.

\section{$R A P D$}

A. fumigatus DNA was prepared as described previously [34]. Fingerprinting of $A$. fumigatus was performed with a single primer NS3: 5' GCAAGTCTGGTGCCAGCAGCC 3 ' from the fungal $18 \mathrm{~S}$ rRNA gene under low stringency amplification conditions [35]. The reaction mixture contained the amplification buffer (50 mM Tris- $\mathrm{HCl}, \mathrm{pH} 8.3,50 \mathrm{mM} \mathrm{KCl}$, bovine serum albumin $0.1 \mathrm{mg} / \mathrm{ml}), 3 \mathrm{mM} \mathrm{MgCl}_{2}$, $200 \mu \mathrm{M}$ of each dNTP, 20 pmol primer NS3 (Pharmacia) and $2.5 \mathrm{U}$ Taq Polymerase (Boerhinger) and c. $10 \mathrm{ng}$ of genomic DNA template in a volume of $50 \mu 1$. The reaction conditions were standardised to ensure optimal reproducibility of RAPD profiles in subsequent investigations and to demonstrate the feasability of the RAPD method. The thermocycler used was a Perkin Elmer Gene Amp 9600 and the conditions were as follows: $2 \mathrm{~min}$ of denaturation at $93^{\circ} \mathrm{C}, 40$ cycles of $0.3 \mathrm{~min}$ at $93^{\circ} \mathrm{C}, 1 \mathrm{~min}$ annealing at $40^{\circ} \mathrm{C}$ and $0.5 \mathrm{~min}$ extension at $72^{\circ} \mathrm{C}$ with a final extension of $6 \mathrm{~min}$ at $72^{\circ} \mathrm{C}$. The amplified products $(15 \mu 1)$ were run on agarose $1.5 \%$ gels in TBE buffer (0.09 M Tris-Borate, $0.002 \mathrm{M}$ EDTA, $\mathrm{pH}$ 8) at $50 \mathrm{~V}$ for $3 \mathrm{~h}$. Gels were stained for $1 \mathrm{~h}$ in ethidium bromide $0.5 \mathrm{mg} / \mathrm{ml}$, de-stained for $20 \mathrm{~min}$ and photographed under UV light with Polaroid film. Mol. wts were estimated and RAPD profiles were assigned to isolates that gave patterns with identical bands and were recorded as the same type. Distinctive profiles were designated as RAPD types (Fig. 3).

\section{Data analysis}

To visualise the relationships between types, dendrograms were constructed by the neighbour-joining method [36] with the TREECON-pc version 2.0 software package (Y. Van de Peer, University of Antwerp, Antwerp, Belgium). The principle of this 
a

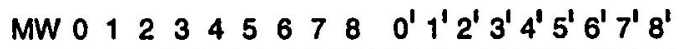

A
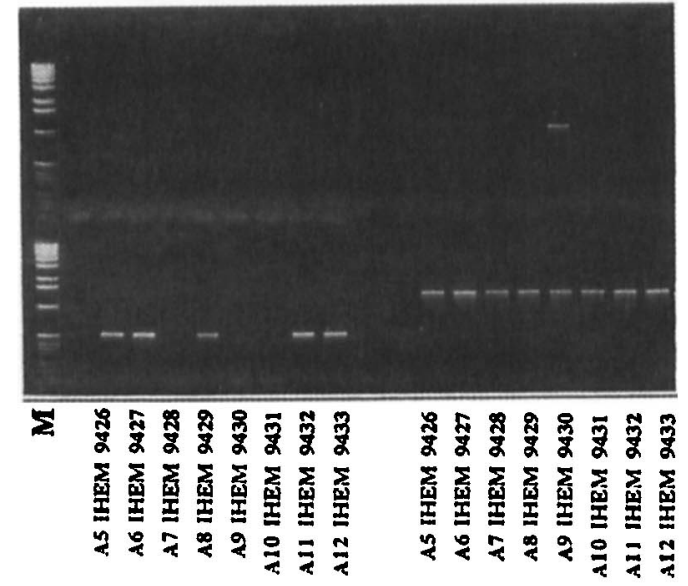

b

MW 0123456789101112131415161718
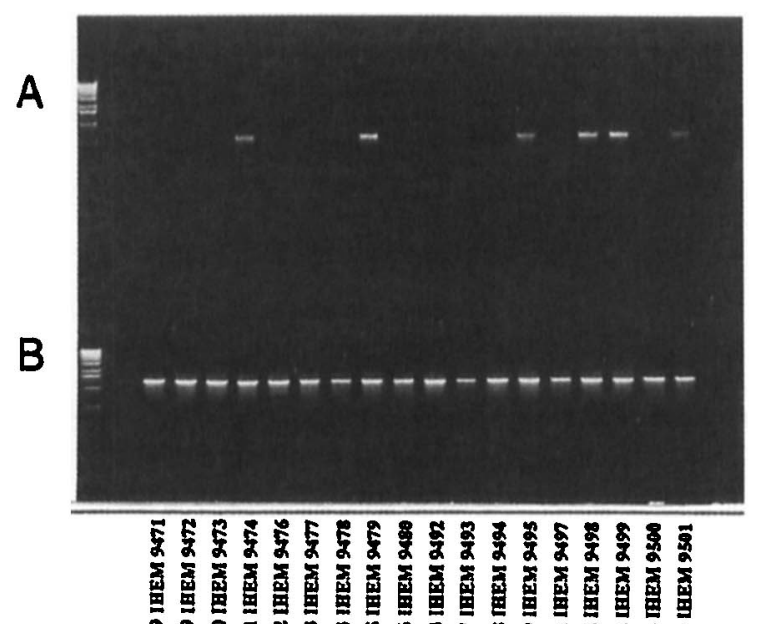

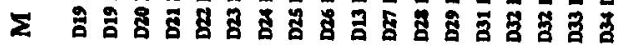

Fig. 2. (a) DNA template of eight clinical strains from patient A (IHEM nos. 9426-9433) amplified with: A the primer pair Afp1/Afp2: SSDP2 (lanes $\mathbf{1}^{\prime}-\mathbf{8}^{\prime}$ ) and B the primer pair Afd1/Afd2 (1-8) and the positive control primer pair Afc1/Afc2 $\left(\mathbf{1}^{\prime}-\mathbf{8}^{\prime}\right)$. Lanes 0 and $0^{\prime}$, negative controls without DNA with Afd1/Afd2 and Afc1/Afc2, respectively. MW, 1-kb ladder marker (Gibco-BRL). (b) DNA template of 18 clinical strains from patient D (IHEM nos. 9471-9480, 9492-9495, 9497-9501) amplified with: $\mathbf{A}$ the primer pair Afd1/Afd2: SSDP3 (1-18) and B the positive control primer pair Afc1/Afc2 (1-18). Lane $\mathbf{0}$, negative control without DNA with Afdl/Afd2 and Afc1/Afc2, respectively. MW, 1-kb ladder marker (Gibco-BRL).

method was to find pairs of types that minimised the total branch length at each stage of clustering, starting with a star-like tree. Unlike other tree-making methods, the neighbour-joining method produced a unique final tree by the principle of minimum evolution and gave the correct tree topology by means of computer simulations. With other tree-making methods, it produces a unique final tree under the principle of minimum evolution and gives the correct tree topology after computer simulations [37]. A. fumigatus types were considered as variants of the same cluster/strain if they differed by one to three genetic loci [38]; they were considered as different clusters/strains if they differed at least by four genetic loci.

\section{Results \\ Reproducibility of each typing method}

To validate the results of the study, different culture conditions were tested to ensure reproducibility. The three typing methods gave clearly reproducible results. Identical results were obtained when isolates were subcultured many times on Sabouraud's medium, when they were cloned, preserved in liquid nitrogen or by lyophilisation and when electrophoresis was performed on samples from every fourth passage of isolates (data not shown).

\section{MLEE}

Of the 11 iso-enzyme activities examined, eight discriminated isolates from the four patients. Isoenzymes AAT, IDH and GDH were monomorphic. All the iso-enzyme patterns and genotypes are summarised in Tables 1,2 and 4-6. Enzyme systems that differentiate isolates are included in Fig. 1. All the isolates examined were differentiated into 33 MLEE types: nine from patient $\mathrm{A}$ isolates, seven from patient $\mathrm{B}$ isolates, seven from patient $\mathrm{C}$ isolates and 14 from patient $\mathrm{D}$ isolates. Four MLEE types were isolated from several patients: MLEE type 1 was isolated from patients $A$ and B (A1M, B8M), MLEE type 2 from patients A and D (A2M, A3M, D1M), MLEE type 9 from patients A and D ( A16M, A17M, A18M, A19M, A20M, D2M), MLEE type 13 from patients B and C (B6M, B5M, C1M). The 33 MLEE types were grouped into seven distinct clusters/strains (Fig. 4a; Tables 4-6).

\section{SSDP}

The combination of the presence (1) or the absence (0) of each single amplified band defined the genotypes (Tables 1, 2 and 4-6). Some of these are represented in Fig. 2. The five examined SSDPs discriminated isolates from three of the four patients. The set of five primers gave 19 SSDP types: 10 from patient A, 6 from patient $B, 1$ from patient $C$ and 14 from patient $D$. Some SSDP types were isolated from different patients, e.g., SSDP type 28 corresponded to isolates from patients A (A4, A11, A20), B (B1) and D (D8, D16, D18, D21, D35). The 19 SSDP types were grouped into six different strains (Fig. 4b, Tables 4-6).

\section{$R A P D$}

The use of primer NS3 resulted in RAPD patterns that were sufficiently polymorphic to differentiate isolates from three patients (A, B and D). Faint bands appeared 


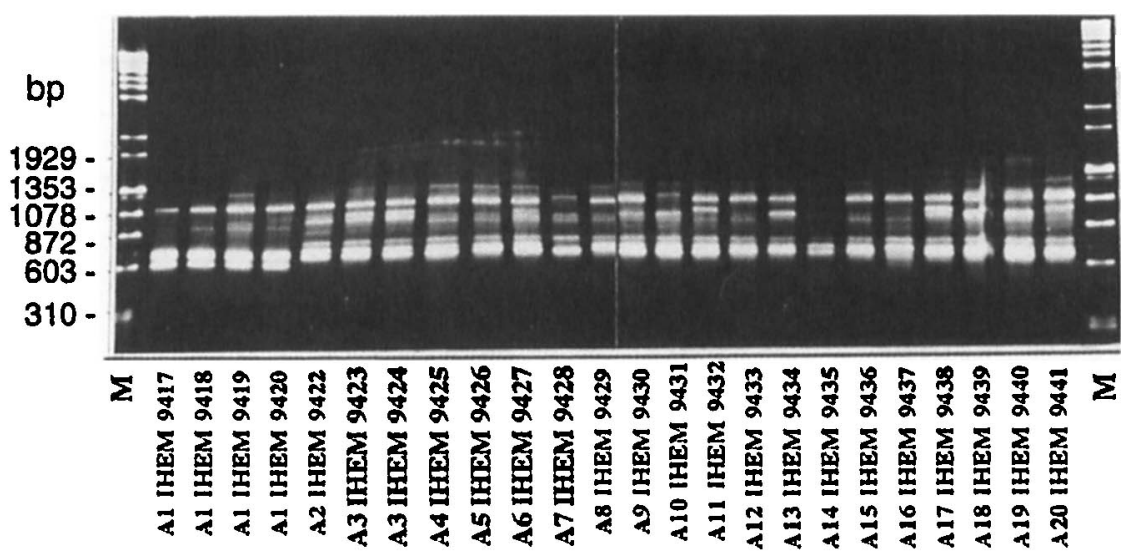

Fig. 3. RAPD typing with primer NS3. Samples were analysed in agarose $1.5 \%$ gel run in TBE buffer. Marker is a mix of $\lambda$ DNA Bst EII digest (Biolabs). Negative reagent control amplifications without added DNA resulted in no bands. The sizes of scored variable bands are indicated by the arrows.

Table 1. A. fumigatus isolates from patients A and B

\begin{tabular}{|c|c|c|c|c|c|c|c|c|}
\hline \multicolumn{3}{|c|}{ IHEM } & \multirow[b]{2}{*}{ Origin } & \multirow{2}{*}{$\begin{array}{l}\text { Date of } \\
\text { isolation }\end{array}$} & \multicolumn{4}{|c|}{ Type } \\
\hline Patient* & Isolate $^{\dagger}$ & Infection & & & MLEE & SSDP & RAPD & Overall \\
\hline \multirow[t]{24}{*}{ A } & 9417 & IA & Broncho-alveolar lavage & $13 / 01 / 95$ & 1 & 7 & 1 & Al \\
\hline & 9418 & IA & Broncho-aspiration & $13 / 01 / 95$ & 1 & 7 & 1 & \\
\hline & 9419 & IA & Broncho-aspiration & $13 / 01 / 95$ & 1 & 7 & 1 & \\
\hline & 9420 & IA & Broncho-aspiration & $13 / 01 / 95$ & 1 & 7 & 1 & \\
\hline & 9422 & IA & Haematology service air & $13 / 01 / 95$ & 2 & 22 & 3 & A2 \\
\hline & 9423 & IA & Haematology service air & $13 / 01 / 95$ & 2 & 17 & 3 & A3 \\
\hline & 9424 & IA & Haematology service air & $23 / 01 / 95$ & 2 & 17 & 3 & \\
\hline & 9425 & IA & Haematology service air & $23 / 01 / 95$ & 3 & 28 & 2 & A4 \\
\hline & 9426 & IA & House drawing-room radiator & $23 / 01 / 95$ & 4 & 12 & 2 & A5 \\
\hline & 9427 & IA & House drawing-room radiator & $23 / 01 / 95$ & 5 & 4 & 3 & A6 \\
\hline & 9428 & IA & House drawing-room door & $23 / 01 / 95$ & 6 & 8 & 4 & A7 \\
\hline & 9429 & IA & House drawing room door & $23 / 01 / 95$ & 6 & 16 & 2 & A8 \\
\hline & 9430 & IA & House bathroom door & $23 / 01 / 95$ & 7 & 15 & 2 & A9 \\
\hline & 9431 & IA & House bathroom door & $23 / 01 / 95$ & 7 & 7 & 3 & A 10 \\
\hline & 9432 & IA & House bathroom door & $23 / 01 / 95$ & 7 & 28 & 3 & A11 \\
\hline & 9433 & IA & House bathroom door & $23 / 01 / 95$ & 7 & 4 & 2 & $\mathrm{~A} 12$ \\
\hline & 9434 & IA & House bathroom door & $23 / 01 / 95$ & 7 & 8 & 2 & A13 \\
\hline & 9435 & IA & House bathroom rack & $23 / 01 / 95$ & 8 & 8 & 6 & A14 \\
\hline & 9436 & IA & House bathroom rack & $23 / 01 / 95$ & 8 & 4 & 2 & A15 \\
\hline & 9437 & IA & House corridor cupboard & $23 / 01 / 95$ & 9 & 15 & 6 & A16 \\
\hline & 9438 & IA & House corridor cupboard & $23 / 01 / 95$ & 9 & 18 & 3 & A17 \\
\hline & 9439 & IA & House corridor cupboard & $23 / 01 / 95$ & 9 & 8 & 2 & A18 \\
\hline & 9440 & IA & House corridor cupboard & $23 / 01 / 95$ & 9 & 22 & 2 & A19 \\
\hline & 9441 & IA & House corridor cupboard & $23 / 01 / 95$ & 9 & 28 & 7 & A20 \\
\hline \multirow[t]{10}{*}{ B } & 9613 & $\mathrm{Col}$ & Broncho-aspiration & $21 / 04 / 95$ & 22 & 28 & 3 & $\mathrm{~B} 1$ \\
\hline & 9614 & $\mathrm{Col}$ & Broncho-aspiration & $21 / 04 / 95$ & 10 & 32 & 7 & $\mathrm{~B} 2$ \\
\hline & 9615 & Col & Broncho-aspiration & $21 / 04 / 95$ & 11 & 24 & 7 & B3 \\
\hline & 9606 & Col & Patient hospital bedroom & $24 / 04 / 95$ & 12 & 26 & 3 & B4 \\
\hline & 9607 & $\mathrm{Col}$ & Patient hospital bedroom & $24 / 04 / 95$ & 13 & 30 & 3 & B5 \\
\hline & 9608 & $\mathrm{Col}$ & Patient hospital bedroom & $24 / 04 / 95$ & 13 & 30 & 2 & B6 \\
\hline & 9609 & $\mathrm{Col}$ & Patient hospital bedroom & $24 / 04 / 95$ & 13 & 30 & 3 & B5 \\
\hline & 9610 & $\mathrm{Col}$ & Patient hospital bedroom & $24 / 04 / 95$ & 14 & 30 & 7 & B7 \\
\hline & 9611 & $\mathrm{Col}$ & Patient hospital bedroom & $24 / 04 / 95$ & 22 & 28 & 3 & B1 \\
\hline & 9612 & Col & Patient hospital bedroom & $24 / 04 / 95$ & 1 & 20 & 3 & B8 \\
\hline
\end{tabular}

IA, invasive aspergillosis; Col, colonisation.

* Patient A, VIZ GIN, invasive aspergillosis (29/11/48, Grenoble, France) suffered from lymphoma after a bone marrow transplant; patient B, AUP HER, bronchopulmonary colonisation $(29 / 06 / 70$, Grenoble, France) was hospitalised for a lung transplant.

†Samples including different colonies were separated.

${ }^{\ddagger}$ Results of four independent experiments for each analysis.

sometimes in the gels after repeated amplifications. However, only clear and reproducible consistent bands were counted and considered to define the isolate genotypes (Fig. 4c, Tables 1, 2 and 4-6). The application of this primer permitted the differentiation of all the isolates into 12 RAPD types: six from patient $A$, three from patient $B$, one from patient $C$ and eight from patient $D$. Some RAPD types were isolated from several patients, e.g., RAPD type 3 was isolated from all patients, RAPD types 2 and 6 were isolated from 
Table 2. A. fumigatus isolates from patients $\mathrm{C}$ and $\mathrm{D}$

\begin{tabular}{|c|c|c|c|c|c|c|c|c|}
\hline \multicolumn{3}{|c|}{ IHEM } & \multirow[b]{2}{*}{ Origin } & \multirow{2}{*}{$\begin{array}{l}\text { Date of } \\
\text { isolation }\end{array}$} & \multicolumn{4}{|c|}{ Type $^{\ddagger}$} \\
\hline Patient* & Isolate $^{\dagger}$ & Infection & & & MLEE & SSDP & RAPD & Overall \\
\hline \multirow[t]{15}{*}{ C } & 9010 & IA & Abdominal drain & $27 / 01 / 94$ & 13 & 30 & 3 & $\mathrm{C} 1$ \\
\hline & 9011 & IA & Abdominal drain & $27 / 01 / 94$ & 13 & 30 & 3 & \\
\hline & 9012 & IA & Abdominal drain & $27 / 01 / 94$ & 13 & 30 & 3 & \\
\hline & 9013 & IA & Abdominal drain & $27 / 01 / 94$ & 13 & 30 & 3 & \\
\hline & 9014 & IA & Abdominal drain & $27 / 01 / 94$ & 13 & 30 & 3 & \\
\hline & 9015 & IA & Hepatic artery & $28 / 01 / 94$ & 15 & 30 & 3 & $\mathrm{C} 2$ \\
\hline & 9016 & IA & Hepatic hilum & $02 / 02 / 94$ & 15 & 30 & 3 & \\
\hline & 9017 & IA & Hepatic hilum & $02 / 02 / 94$ & 15 & 30 & 3 & \\
\hline & 9018 & IA & Hepatic hilum & $02 / 02 / 94$ & 15 & 30 & 3 & \\
\hline & 9019 & IA & Liver & $02 / 02 / 94$ & 15 & 30 & 3 & \\
\hline & 9020 & IA & Bile & $02 / 02 / 94$ & 16 & 30 & 3 & $\mathrm{C} 3$ \\
\hline & 9021 & IA & Lung (autopsy) & $07 / 02 / 94$ & 17 & 30 & 3 & $\mathrm{C} 4$ \\
\hline & 9022 & IA & Hepatic artery (autopsy) & $07 / 02 / 94$ & 18 & 30 & 3 & C5 \\
\hline & 9023 & IA & Portal vein (autopsy) & $07 / 02 / 94$ & 19 & 30 & 3 & $\mathrm{C} 6$ \\
\hline & 9024 & IA & Hepatic hilum (autopsy) & $07 / 02 / 94$ & 20 & 30 & 3 & $\mathrm{C} 7$ \\
\hline \multirow[t]{42}{*}{ D } & 9393 & Col & Broncho-alveolar lavage & $15 / 12 / 94$ & 2 & 17 & 2 & D1 \\
\hline & 9394 & Col & Broncho-alveolar lavage & $15 / 12 / 94$ & 9 & 22 & 9 & D2 \\
\hline & 9395 & $\mathrm{Col}$ & Broncho-alveolar lavage & $15 / 12 / 94$ & 23 & 6 & 6 & D3 \\
\hline & 9396 & Col & Broncho-alveolar lavage & $15 / 12 / 94$ & 23 & 6 & 6 & \\
\hline & 9397 & Col & Broncho-alveolar lavage & $15 / 12 / 94$ & 32 & 23 & 6 & D4 \\
\hline & 9453 & IA & Broncho-aspiration & $28 / 12 / 94$ & 21 & 20 & 9 & D5 \\
\hline & 9454 & IA & Broncho-aspiration & $28 / 12 / 94$ & 22 & 24 & 9 & D6 \\
\hline & 9455 & IA & Broncho-aspiration & $28 / 12 / 94$ & 22 & 16 & 6 & D7 \\
\hline & 9456 & IA & Broncho-aspiration & $28 / 12 / 94$ & 21 & 28 & 2 & D8 \\
\hline & 9457 & IA & Broncho-aspiration & $28 / 12 / 94$ & 21 & 24 & 6 & D9 \\
\hline & 9458 & IA & Broncho-alveolar lavage & $10 / 01 / 95$ & 23 & 30 & 2 & D10 \\
\hline & 9459 & IA & Broncho-alveolar lavage & $10 / 01 / 95$ & 23 & 6 & 2 & D11 \\
\hline & 9460 & IA & Broncho-alveolar lavage & $10 / 01 / 95$ & 26 & 4 & 2 & D12 \\
\hline & 9461 & IA & Broncho-alveolar lavage & $10 / 01 / 95$ & 23 & 22 & 2 & D13 \\
\hline & 9462 & IA & Broncho-alveolar lavage & $10 / 01 / 95$ & 23 & 30 & 2 & D10 \\
\hline & 9463 & IA & Broncho-aspiration & $10 / 01 / 95$ & 23 & 22 & 2 & D14 \\
\hline & 9464 & IA & Sputum & $11 / 01 / 95$ & 25 & 19 & 2 & D15 \\
\hline & 9465 & IA & Sputum & $11 / 01 / 95$ & 26 & 4 & 2 & D12 \\
\hline & 9466 & IA & Sputum & $11 / 01 / 95$ & 27 & 28 & 2 & D16 \\
\hline & 9467 & IA & Sputum & $11 / 01 / 95$ & 26 & 20 & 6 & D17 \\
\hline & 9468 & IA & Sputum & $11 / 01 / 95$ & 26 & 4 & 2 & D12 \\
\hline & 9469 & IA & Broncho-alveolar lavage & $12 / 01 / 95$ & 28 & 28 & 10 & D18 \\
\hline & 9470 & IA & Broncho-alveolar lavage & $15 / 01 / 95$ & 28 & 24 & 6 & D19 \\
\hline & 9471 & IA & Broncho-alveolar lavage & $15 / 01 / 95$ & 28 & 24 & 6 & \\
\hline & 9472 & IA & Broncho-alveolar lavage & $15 / 01 / 95$ & 28 & 24 & 6 & \\
\hline & 9473 & IA & Broncho-alveolar lavage & $15 / 01 / 95$ & 28 & 8 & 6 & D20 \\
\hline & 9474 & IA & Broncho-alveolar lavage & $15 / 01 / 95$ & 27 & 28 & 10 & D21 \\
\hline & 9476 & IA & Broncho-aspiration & $16 / 01 / 95$ & 23 & 30 & 11 & $\mathrm{D} 22$ \\
\hline & 9477 & IA & Broncho-aspiration & $16 / 01 / 95$ & 28 & 16 & 12 & D23 \\
\hline & 9478 & IA & Broncho-aspiration & $16 / 01 / 95$ & 28 & 8 & 12 & D24 \\
\hline & 9479 & IA & Broncho-aspiration & $16 / 01 / 95$ & 26 & 20 & 13 & D25 \\
\hline & 9480 & IA & Broncho-aspiration & $16 / 01 / 95$ & 28 & 24 & 13 & $\mathrm{D} 26$ \\
\hline & 9492 & IA & Broncho-alveolar lavage & $16 / 01 / 95$ & 23 & 22 & 2 & D13 \\
\hline & 9493 & IA & Broncho-alveolar lavage & $16 / 01 / 95$ & 29 & 24 & 2 & $\mathrm{D} 27$ \\
\hline & 9494 & IA & Broncho-alveolar lavage & $16 / 01 / 95$ & 33 & 30 & 2 & D28 \\
\hline & 9495 & IA & Broncho-alveolar lavage & $16 / 01 / 95$ & 30 & 25 & 2 & D29 \\
\hline & 9496 & IA & Broncho-alveolar lavage & $16 / 01 / 95$ & 22 & 24 & 14 & $\mathrm{D} 30$ \\
\hline & 9497 & IA & Broncho-aspiration & $18 / 01 / 95$ & 31 & 20 & 2 & D31 \\
\hline & 9498 & IA & Broncho-aspiration & $18 / 01 / 95$ & 31 & 20 & 3 & D32 \\
\hline & 9499 & IA & Broncho-aspiration & $18 / 01 / 95$ & 31 & 20 & 1 & D33 \\
\hline & 9500 & IA & Broncho-aspiration & $18 / 01 / 95$ & 22 & 32 & 9 & D34 \\
\hline & 9501 & IA & Broncho-aspiration & $18 / 01 / 95$ & 26 & 28 & 9 & D35 \\
\hline
\end{tabular}

IA, invasive aspergillosis; Col, colonisation.

* Patient C, MAG AMB, invasive aspergillosis (16/06/93, Milan, Italy) was hospitalised for a liver transplant; patient D, TAK REB, invasive aspergillosis following bronchopulmonary colonisation (14/03/59, Lyon, France) was hospitalised for a lung transplant.

†Samples including different colonies were separated.

${ }^{\ddagger}$ Results of four independent experiments for each analysis.

patients A and D. The 12 RAPD types were grouped into four different strains (Fig. 4c, Tables 4-6).

\section{Combination of methods}

When MLEE, SSDP and RAPD analysis were combined, 69 electrophoretic types (ETs) clustered into 15 clusters/strains were defined from the 91 isolates (Tables 1, 2 and 4-6 Fig. 4d). Inside clusters, ETs were considered as variants of the same strain. On average strains were composed of four variants (extremes 2-10). The discrimination power was significantly increased when typing methods were combined in pairs. Moreover, the three combined 
Table 3. Origin and sequences of SSDPs

\begin{tabular}{|c|c|c|c|}
\hline RAPD primer & SSDP marker* & SSDP primer & Sequence $^{\dagger}$ \\
\hline OpQ6 & SSDP1 & $\begin{array}{l}\text { Afp1 } \\
\text { Afp2 }\end{array}$ & $\begin{array}{l}\text { TTGGGGAGATTACCGAACTGG } \\
\text { CCTTGACAACCGTCCCATTTC }\end{array}$ \\
\hline OPAj12 & SSDP2 & $\begin{array}{l}\text { AfMp1 } \\
\text { AfMp2 }\end{array}$ & $\begin{array}{l}\text { CAGTTCCCGTCTTGACCTC } \\
\text { CAGTTCCCGTTTTCCTAGTA }\end{array}$ \\
\hline R108 & $\begin{array}{l}\text { SSDP3 } \\
\text { SSDP4 } \\
\text { SSDP5 }\end{array}$ & $\begin{array}{l}\text { Afd1 } \\
\text { Afd2 } \\
\text { Afs4 } \\
\text { Afr4 } \\
\text { Afs5 } \\
\text { Afr5 }\end{array}$ & $\begin{array}{l}\text { GTATTGCCCTATAACTTCTT } \\
\text { GTATTGCCCTATTCCCAAAG } \\
\text { GTATTGCCCTAGCTTACTAA } \\
\text { GTATTGCCCTATTACTAAAG } \\
\text { GTATTGCCCTAAGGATTCTA } \\
\text { GTATTGCCCTAGCTTGCTAA }\end{array}$ \\
\hline
\end{tabular}

typing methods gave a higher degree of discrimination; each of the three typing methods complemented each other to give a better definition of strain and variants.

\section{Epidemiological analysis}

Each typing method demonstrated that the four isolates infecting patient A were identical (MLEE type 1, SSDP type 7, RAPD type 1) and combining the three methods were labelled A1 ET. This patient was infected by a single strain. However, the uniqueness of the type corresponding to infecting isolates from patient A with IA may be the result of the limited number of bronchopulmonary samples tested. The environmental isolates corresponded to eight distinct MLEE types clustered into two MLEE strains (VI and VII) distinct from the clinical strain (Fig. 4a). These isolates corresponded to 10 SSDP types clustered into four distinct SSDP strains (III-VI) (Fig. 4b). The A1 clinical type and A10 environmental type were identical by this typing method. These isolates corresponded to five RAPD types grouped into four distinct RAPD strains (Fig. 4c). The clinical isolates belonged to the IV RAPD strain as A16 and A14 environmental isolates. With the three combined methods (Fig. 4d), it was concluded that the clinical isolate A1 belongs to strain II and the environmental isolates defined as 19 distinct ETs are clustered in six strains (X-XV).

Genotypes B1, B2 and B3 from patient B corresponded to clinical isolates. They corresponded to three distinct MLEE strains (Fig. 4a), two distinct SSDP strains (Fig. 4b) and one RAPD strain (Fig. 4c). When the three methods were combined, B1, B2 and B3 corresponded to two distinct strains (Fig. 4d). B2 and B3 are variants of the strain I; therefore, this patient was infected by two distinct strains. The three methods were concordant in that B1 clinical ET was also found in the environment of this patient (Table 4). Other environmental isolates clustered in three MLEE strains, two SSDP strains and two RAPD strains; when the three methods were combined, they finally clustered in four strains (Fig. 4d).
Only the MLEE method revealed the polymorphism of the samples from patient C. Neither SSDP nor RAPD could demonstrate differences; thus, the isolates clustered in four MLEE strains, one SSDP strain and one RAPD strain. Combining the three methods led to the conclusion that patient $\mathrm{C}$ was infected by three distinct strains in different body locations (Fig. 4d). Strain V was composed of two variants, strain VI of three variants. These two strains were patient C ETspecific. Strain VII comprised 10 variants, among them only two correspond to patient C ETs (Fig.4d).

When D1-D4 were isolated, patient D suffered from bronchopulmonary colonisation by $A$. fumigatus. These ETs corresponded to three MLEE strains (Fig. 4a), three SSDP strains (Fig. 4b) and two RAPD strains (Fig. 4c). Combining the three methods, these ETs were clustered in four strains (Fig. 4d). D5-D34 ETs were isolated when patient D suffered from IA. These ETs corresponded to four MLEE strains, but D1 and D2 related strains were not found during the IA infection. They corresponded to six SSDP strains and to three RAPD strains. When the three methods were combined, these ETs were grouped in seven strains and D1 and D2 related strains were not found during the IA. It is concluded that the bronchopulmonary infection was caused by four distinct strains. Two of these strains were not isolated later. The other two persisted and may have differentiated: strain VII first contained D3 variant and then five other variants (D10, D11, D13, D14, D22); also strain VIII first contained D4 variant and then four other variants (D12, D17, D24, D35). In total, patient $\mathrm{D}$ was infected by nine distinct strains. Moreover, patient D isolates illustrated the great diversity of this pathogen (Fig. 4d).

\section{Discussion}

In this study, $91 \mathrm{~A}$. fumigatus isolates were analysed by three typing methods - MLEE, RAPD and SSDP each applied in different EBGA network laboratories. The three combined typing methods demonstrated 
Table 4. Combining profiles of A. fumigatus isolates from patients A and B obtained by MLEE, SSDP and RAPD analysis

\begin{tabular}{|c|c|c|c|c|c|c|c|c|c|c|c|c|c|c|c|c|c|c|c|c|c|c|c|c|c|}
\hline \multirow[b]{2}{*}{ Patient* } & \multirow[b]{2}{*}{ Isolate $^{\dagger}$} & \multicolumn{9}{|c|}{$\begin{array}{c}\text { MLEE } \\
\text { Alleles at the indicated enzymic locus }{ }^{\ddagger}\end{array}$} & \multicolumn{6}{|c|}{$\begin{array}{c}\text { SSDP } \\
\text { SSDPs used (scored band) } \S\end{array}$} & \multicolumn{8}{|c|}{$\begin{array}{c}\text { RAPD } \\
\text { Scored band with size }(\mathrm{kb})^{\S}\end{array}$} & \multirow{2}{*}{$\begin{array}{l}\text { Overall } \\
\text { Type }\end{array}$} \\
\hline & & PepA & PepB & GPI & PGM & HK & ME & NP & PK & Type & S1 & $\mathrm{S} 2$ & S3 & S4 & S5 & Type & 0.6 & 0.65 & 0.7 & 0.78 & 0.95 & 1 & 1.1 & Type & \\
\hline \multirow[t]{24}{*}{$\overline{\mathrm{A}}$} & $9417 \mathrm{c}$ & 2 & $1 / 2$ & 2 & 1 & 1 & 1 & 1 & 1 & 1 & 0 & 0 & 0 & 0 & 1 & 7 & 1 & 1 & 0 & 0 & 0 & 0 & 1 & 1 & $\mathrm{~A} 1$ \\
\hline & $9418 \mathrm{c}$ & 2 & $1 / 2$ & 2 & 1 & 1 & 1 & 1 & 1 & 1 & 0 & 0 & 0 & 0 & 1 & 7 & 1 & 1 & 0 & 0 & 0 & 0 & 1 & 1 & \\
\hline & $9419 \mathrm{c}$ & 2 & $1 / 2$ & 2 & 1 & 1 & 1 & 1 & 1 & 1 & 0 & 0 & 0 & 0 & 1 & 7 & 1 & 1 & 0 & 0 & 0 & 0 & 1 & 1 & \\
\hline & $9420 \mathrm{c}$ & 2 & $1 / 2$ & 2 & 1 & 1 & 1 & 1 & 1 & 1 & 0 & 0 & 0 & 0 & 1 & 7 & 1 & 1 & 0 & 0 & 0 & 0 & 1 & 1 & \\
\hline & $9422 \mathrm{e}$ & 1 & 1 & 1 & 1 & 1 & 2 & 1 & 1 & 2 & 1 & 0 & 0 & 0 & 1 & 22 & 0 & 1 & 1 & 1 & 1 & 1 & 1 & 3 & $\mathrm{~A} 2$ \\
\hline & $9423 \mathrm{e}$ & 1 & 1 & 1 & 1 & 1 & 2 & 1 & 1 & 2 & 1 & 1 & 1 & 0 & 1 & 17 & 0 & 1 & 1 & 1 & 1 & 1 & 1 & 3 & A3 \\
\hline & $9424 \mathrm{e}$ & 1 & 1 & 1 & 1 & 1 & 2 & 1 & 1 & 2 & 1 & 1 & 1 & 0 & 1 & 17 & 0 & 1 & 1 & 1 & 1 & 1 & $i$ & 3 & \\
\hline & $9425 \mathrm{e}$ & 1 & 2 & 1 & 2 & 2 & 2 & 1 & 1 & 3 & 0 & 0 & 1 & 1 & 1 & 28 & 0 & 1 & 1 & 1 & 1 & 0 & 1 & 2 & A4 \\
\hline & $9426 \mathrm{e}$ & 1 & 1 & 1 & 1 & 1 & 2 & 2 & 1 & 4 & 0 & 0 & 1 & i & 0 & 12 & 0 & 1 & 1 & 1 & 1 & 0 & 1 & 2 & A5 \\
\hline & $9427 \mathrm{e}$ & 2 & 2 & 1 & 1 & 1 & 2 & 2 & 1 & 5 & 0 & 0 & 1 & 0 & 0 & 4 & 0 & 1 & 1 & 1 & 1 & 1 & 1 & 3 & A6 \\
\hline & $9428 \mathrm{e}$ & 1 & $1 / 2$ & 1 & 1 & 2 & 2 & 2 & 1 & 6 & 0 & 0 & 0 & 0 & 0 & 8 & 0 & 1 & 0 & 1 & 1 & 0 & 1 & 4 & A7 \\
\hline & $9429 \mathrm{e}$ & 1 & $1 / 2$ & 1 & 1 & 2 & 2 & 2 & 1 & 6 & 0 & 0 & 0 & 1 & 0 & 16 & 0 & 1 & 1 & 1 & 1 & 0 & i & 2 & A8 \\
\hline & $9430 \mathrm{e}$ & 2 & 1 & 1 & 1 & 2 & 2 & 1 & 1 & 7 & 0 & 1 & 0 & 1 & 0 & 15 & 0 & 1 & 1 & 1 & 1 & 0 & 1 & 2 & A9 \\
\hline & $9431 \mathrm{e}$ & 2 & 1 & 1 & 1 & 2 & 2 & 1 & 1 & 7 & 0 & 0 & 0 & 0 & 1 & 7 & 0 & 1 & 1 & 1 & 1 & 1 & 1 & 3 & $\mathrm{~A} 10$ \\
\hline & $9432 \mathrm{e}$ & 2 & 1 & 1 & 1 & 2 & 2 & 1 & 1 & 7 & 0 & 0 & 1 & 1 & 1 & 28 & 0 & 1 & 1 & 1 & 1 & 1 & $i$ & 3 & Al1 \\
\hline & $9433 \mathrm{e}$ & 2 & 1 & 1 & 1 & 2 & 2 & 1 & 1 & 7 & 0 & 0 & 1 & 0 & 0 & 4 & 0 & 1 & 1 & 1 & 0 & 0 & 1 & 6 & $\mathrm{~A} 12$ \\
\hline & $9434 \mathrm{e}$ & 2 & 1 & 1 & 1 & 2 & 2 & 1 & 1 & 7 & 0 & 0 & 0 & 0 & 0 & 8 & 0 & 1 & 1 & 1 & 1 & 0 & 1 & 2 & $\mathrm{~A} 13$ \\
\hline & $9435 \mathrm{e}$ & 1 & 1 & 1 & 2 & 2 & 2 & 1 & 1 & 8 & 0 & 0 & 0 & 0 & 0 & 8 & 0 & 1 & 1 & 1 & 0 & 0 & $i$ & 6 & Al4 \\
\hline & $9436 \mathrm{e}$ & 1 & 1 & 1 & 2 & 2 & 2 & 1 & 1 & 8 & 0 & 0 & 1 & 0 & 0 & 4 & 0 & 1 & 1 & 1 & 0 & 0 & $i$ & 6 & A15 \\
\hline & $9437 \mathrm{e}$ & 1 & 2 & 1 & 2 & 2 & 2 & 2 & 2 & 9 & 0 & 1 & 0 & 1 & 0 & 15 & 0 & 1 & 1 & 1 & 0 & 0 & i & 6 & A16 \\
\hline & $9438 \mathrm{e}$ & $i$ & 2 & 1 & 2 & 2 & 2 & 2 & 2 & 9 & 1 & 0 & 1 & 0 & 1 & 18 & 0 & 1 & 1 & 1 & 1 & 1 & $i$ & 3 & A17 \\
\hline & $9439 \mathrm{e}$ & 1 & 2 & 1 & 2 & 2 & 2 & 2 & 2 & 9 & 0 & 0 & 0 & 0 & 0 & 8 & 0 & 1 & 1 & 1 & 1 & 0 & 1 & 2 & A18 \\
\hline & $9440 \mathrm{e}$ & 1 & 2 & 1 & 2 & 2 & 2 & 2 & 2 & 9 & 1 & 0 & 0 & 0 & 1 & 22 & 0 & 1 & 1 & 1 & 1 & 0 & 1 & 2 & A19 \\
\hline & $9441 \mathrm{e}$ & 1 & 2 & $i$ & 2 & 2 & 2 & 2 & 2 & 9 & 0 & 0 & 1 & 1 & 1 & 28 & 0 & 1 & 1 & 1 & 0 & 1 & 1 & 7 & A20 \\
\hline \multirow{10}{*}{ B } & $9613 \mathrm{c}$ & 2 & 1 & 2 & 1 & 1 & 1 & 1 & 1 & 22 & 0 & 0 & 1 & 1 & 1 & 28 & 0 & 1 & 1 & 1 & 1 & 1 & 1 & 3 & B1 \\
\hline & $9614 \mathrm{c}$ & 2 & 1 & 2 & 1 & 2 & 1 & 2 & 1 & 10 & 0 & 0 & 0 & 1 & 1 & 32 & 0 & 1 & 1 & 1 & 0 & 1 & 1 & 7 & B2 \\
\hline & $9615 \mathrm{c}$ & 2 & $1 / 2$ & 2 & 1 & 2 & 1 & 1 & 2 & 11 & 1 & 0 & 1 & 1 & 1 & 24 & 0 & 1 & 1 & 1 & 0 & 1 & $i$ & 7 & B3 \\
\hline & $9606 \mathrm{e}$ & 2 & $1 / 2$ & 1 & 1 & 1 & 2 & 1 & 1 & 12 & 1 & 0 & 0 & 1 & 1 & 26 & 0 & 1 & 1 & 1 & 1 & 1 & 1 & 3 & B4 \\
\hline & $9607 \mathrm{e}$ & 1 & $1 / 2$ & 2 & 1 & $1 / 2$ & 1 & 2 & 1 & 13 & 1 & 0 & 0 & 1 & 1 & 30 & 0 & 1 & $i$ & 1 & 1 & 1 & 1 & 3 & B5 \\
\hline & $9608 \mathrm{e}$ & 1 & $1 / 2$ & 2 & 1 & $1 / 2$ & 1 & 2 & 1 & 13 & 1 & 0 & 0 & 1 & 1 & 30 & 0 & 1 & $i$ & 1 & 1 & 0 & 1 & 2 & B6 \\
\hline & $9609 \mathrm{e}$ & 1 & $1 / 2$ & 2 & 1 & $1 / 2$ & 1 & 2 & 1 & 13 & 1 & 0 & 0 & 1 & 1 & 30 & 0 & 1 & 1 & 1 & 1 & 1 & 1 & 3 & B5 \\
\hline & $9610 \mathrm{e}$ & 2 & $1 / 2$ & 1 & 2 & 2 & 1 & 2 & 1 & 14 & 1 & 0 & 0 & 1 & 1 & 30 & 0 & 1 & 1 & 1 & 0 & 1 & 1 & 7 & B7 \\
\hline & $9611 \mathrm{e}$ & 2 & 1 & 2 & 1 & 1 & 1 & 1 & I & 22 & 0 & 0 & 1 & 1 & 1 & 28 & 0 & 1 & 1 & 1 & 1 & 1 & 1 & 3 & B1 \\
\hline & $9612 \mathrm{e}$ & 2 & $1 / 2$ & 2 & 1 & 1 & 1 & 1 & 1 & 1 & 0 & 0 & 1 & 0 & 1 & 20 & 0 & 1 & 1 & 1 & 1 & 1 & $i$ & 3 & B8 \\
\hline
\end{tabular}


Table 5. Combining profiles of A. fumigatus isolates from patients C and D obtained by MLEE, SSDP and RAPD analysis*

\begin{tabular}{|c|c|c|c|c|c|c|c|c|c|c|c|c|c|c|c|c|c|c|c|c|c|c|c|c|c|}
\hline \multirow[b]{2}{*}{ Patient* } & \multirow[b]{2}{*}{ Isolate $^{\dagger}$} & \multicolumn{9}{|c|}{$\begin{array}{c}\text { MLEE } \\
\text { Alleles at the indicated enzymic locus }{ }^{\ddagger}\end{array}$} & \multicolumn{6}{|c|}{$\begin{array}{c}\text { SSDP } \\
\text { SSDPs used (scored band) }{ }^{\S}\end{array}$} & \multicolumn{8}{|c|}{$\begin{array}{c}\text { RAPD } \\
\text { Scored band with size }(\mathbf{k b})^{\S}\end{array}$} & \multirow{2}{*}{$\begin{array}{l}\text { Overall } \\
\text { Type }\end{array}$} \\
\hline & & PepA & PepB & GPI & PGM & $\mathrm{HK}$ & $\mathrm{ME}$ & NP & $\mathrm{PK}$ & Type & S1 & S2 & $\mathrm{S3}$ & S4 & S5 & Type & 0.6 & 0.65 & 0.7 & 0.78 & 0.95 & 1 & 1.1 & Type & \\
\hline \multirow[t]{15}{*}{$\bar{C}$} & 9010 & 1 & $1 / 2$ & 2 & 1 & $1 / 2$ & 1 & 2 & 1 & 13 & 1 & 0 & 0 & 1 & 1 & 30 & 0 & 1 & 1 & 1 & 1 & 1 & 1 & 3 & $\mathrm{Cl}$ \\
\hline & 9011 & 1 & $1 / 2$ & 2 & 1 & $1 / 2$ & 1 & 2 & 1 & 13 & 1 & 0 & 0 & 1 & 1 & 30 & 0 & 1 & 1 & 1 & 1 & 1 & 1 & 3 & \\
\hline & 9012 & 1 & $1 / 2$ & 2 & 1 & $1 / 2$ & 1 & 2 & 1 & 13 & 1 & 0 & 0 & 1 & 1 & 30 & 0 & 1 & 1 & 1 & 1 & 1 & 1 & 3 & \\
\hline & 9013 & 1 & $1 / 2$ & 2 & 1 & $1 / 2$ & 1 & 2 & 1 & 13 & 1 & 0 & 0 & 1 & 1 & 30 & 0 & 1 & 1 & 1 & 1 & 1 & 1 & 3 & \\
\hline & 9014 & 1 & $1 / 2$ & 2 & 1 & $1 / 2$ & 1 & 2 & 1 & 13 & 1 & 0 & 0 & 1 & 1 & 30 & 0 & 1 & 1 & 1 & 1 & 1 & 1 & 3 & \\
\hline & 9015 & 2 & 1 & 2 & 1 & 1 & 1 & 2 & 1 & 15 & 1 & 0 & 0 & 1 & 1 & 30 & 0 & 1 & 1 & 1 & 1 & 1 & 1 & 3 & C2 \\
\hline & 9016 & 2 & 1 & 2 & 1 & 1 & 1 & 2 & 1 & 15 & 1 & 0 & 0 & 1 & 1 & 30 & 0 & 1 & 1 & 1 & 1 & 1 & 1 & 3 & \\
\hline & 9017 & 2 & 1 & 2 & 1 & 1 & 1 & 2 & 1 & 15 & 1 & 0 & 0 & 1 & 1 & 30 & 0 & 1 & 1 & 1 & 1 & 1 & 1 & 3 & \\
\hline & 9018 & 2 & 1 & 2 & 1 & 1 & 1 & 2 & 1 & 15 & 1 & 0 & 0 & 1 & 1 & 30 & 0 & 1 & 1 & 1 & 1 & 1 & 1 & 3 & \\
\hline & 9019 & 2 & 1 & 2 & 1 & 1 & 1 & 2 & 1 & 15 & 1 & 0 & 0 & 1 & 1 & 30 & 0 & 1 & 1 & 1 & 1 & 1 & 1 & 3 & \\
\hline & 9020 & 1 & 1 & 2 & 1 & 1 & 1 & 2 & 1 & 16 & 1 & 0 & 0 & 1 & 1 & 30 & 0 & 1 & 1 & 1 & 1 & 1 & 1 & 3 & C3 \\
\hline & 9021 & 2 & 1 & 1 & 1 & 1 & 1 & 1 & 1 & 17 & 1 & 0 & 0 & 1 & 1 & 30 & 0 & 1 & 1 & 1 & 1 & 1 & 1 & 3 & C4 \\
\hline & 9022 & 1 & $1 / 2$ & 1 & 1 & 1 & 1 & 2 & 1 & 18 & 1 & 0 & 0 & 1 & 1 & 30 & 0 & 1 & 1 & 1 & 1 & 1 & 1 & 3 & C5 \\
\hline & 9023 & 2 & $1 / 2$ & 1 & 1 & 1 & 1 & 2 & 1 & 19 & 1 & 0 & 0 & 1 & 1 & 30 & 0 & 1 & 1 & 1 & 1 & 1 & 1 & 3 & C6 \\
\hline & 9024 & 1 & $1 / 2$ & 1 & 1 & $1 / 2$ & 1 & 2 & 1 & 20 & 1 & 0 & 0 & 1 & 1 & 30 & 0 & 1 & 1 & 1 & 1 & 1 & 1 & 3 & C7 \\
\hline \multirow[t]{30}{*}{ D } & $9393 \mathrm{col}$ & 1 & 1 & 1 & 1 & 1 & 2 & 1 & 1 & 2 & 1 & 1 & 1 & 0 & 1 & 17 & 0 & 1 & 1 & 1 & 1 & 0 & 1 & 2 & D1 \\
\hline & $9394 \mathrm{col}$ & 1 & 2 & 1 & 2 & 2 & 2 & 2 & 2 & 9 & 1 & 0 & 0 & 0 & 1 & 22 & 0 & 1 & 1 & 1 & 0 & 0 & 1 & 9 & D2 \\
\hline & $9395 \mathrm{col}$ & 1 & 1 & 2 & 1 & $1 / 2$ & 1 & 2 & 1 & 23 & 1 & 0 & 0 & 0 & 0 & 6 & 0 & 1 & 1 & 1 & 0 & 0 & 1 & 6 & D3 \\
\hline & $9396 \mathrm{col}$ & 1 & 1 & 2 & 1 & $1 / 2$ & 1 & 2 & 1 & 23 & 1 & 0 & 0 & 0 & 0 & 6 & 0 & 1 & 1 & 1 & 0 & 0 & 1 & 6 & \\
\hline & $9397 \mathrm{col}$ & 2 & $1 / 2$ & 2 & 2 & $1 / 2$ & 2 & 2 & 2 & 32 & 0 & 1 & 0 & 0 & 1 & 23 & 0 & 1 & 1 & 1 & 0 & 0 & 1 & 6 & D4 \\
\hline & $9453 \mathrm{ia}$ & 2 & 1 & 2 & 2 & 1 & 1 & 2 & 1 & 21 & 0 & 0 & 1 & 0 & 1 & 20 & 0 & 1 & 1 & 1 & 0 & 0 & 1 & 9 & D5 \\
\hline & $9454 \mathrm{ia}$ & 2 & 1 & 2 & 1 & 1 & 1 & 1 & 1 & 22 & 1 & 0 & 1 & 1 & 1 & 24 & 0 & 1 & 1 & 1 & 0 & 0 & 1 & 9 & D6 \\
\hline & $9455 i a$ & 2 & 1 & 2 & 1 & 1 & 1 & 1 & 1 & 22 & 0 & 0 & 0 & 1 & 0 & 16 & 0 & 1 & 1 & 1 & 0 & 0 & 1 & 6 & D7 \\
\hline & $9456 \mathrm{ia}$ & 2 & 1 & 2 & 2 & 1 & 1 & 2 & 1 & 21 & 0 & 0 & 1 & 1 & 1 & 28 & 0 & 1 & 1 & 1 & 1 & 0 & 1 & 2 & D8 \\
\hline & $9457 \mathrm{ia}$ & 2 & 1 & 2 & 2 & 1 & 1 & 2 & 1 & 21 & 1 & 0 & 1 & 1 & 1 & 24 & 0 & 1 & 1 & 1 & 0 & 0 & 1 & 6 & D9 \\
\hline & $9458 \mathrm{ia}$ & 1 & 1 & 2 & 1 & $1 / 2$ & 1 & 2 & 1 & 23 & 1 & 0 & 0 & 1 & 1 & 30 & 0 & 1 & 1 & 1 & 1 & 0 & 1 & 2 & D10 \\
\hline & 9459ia & 1 & 1 & 2 & 1 & $1 / 2$ & 1 & 2 & 1 & 23 & 1 & 0 & 0 & 0 & 0 & 6 & 0 & 1 & 1 & 1 & 1 & 0 & 1 & 2 & D11 \\
\hline & $9460 \mathrm{ia}$ & 2 & 1 & 1 & 2 & $1 / 2$ & 1 & 2 & 1 & 26 & 0 & 0 & 1 & 0 & 0 & 4 & 0 & 1 & 1 & 1 & 1 & 0 & 1 & 2 & D12 \\
\hline & 9461 ia & 1 & 1 & 2 & 1 & $1 / 2$ & 1 & 2 & 1 & 23 & 1 & 0 & 0 & 0 & 1 & 22 & 0 & 1 & 1 & 1 & 1 & 0 & 1 & 2 & D13 \\
\hline & $9462 \mathrm{ia}$ & 1 & 1 & 2 & 1 & $1 / 2$ & 1 & 2 & 1 & 23 & 1 & 0 & 0 & 1 & 1 & 30 & 0 & 1 & 1 & 1 & 1 & 0 & 1 & 2 & D10 \\
\hline & $9463 i a$ & 1 & 1 & 2 & 1 & $1 / 2$ & 1 & 2 & 1 & 23 & 1 & 0 & 0 & 0 & 1 & 22 & 0 & 1 & 1 & 1 & 1 & 0 & 1 & 2 & DI4 \\
\hline & $9464 \mathrm{ia}$ & $1 / 2$ & 1 & 2 & 1 & 1 & 1 & 1 & 1 & 25 & 0 & 1 & 1 & 0 & 1 & 19 & 0 & 1 & 1 & 1 & 1 & 0 & 1 & 2 & D15 \\
\hline & $9465 \mathrm{ia}$ & 2 & 1 & 1 & 2 & $1 / 2$ & 1 & 2 & 1 & 26 & 0 & 0 & 1 & 0 & 0 & 4 & 0 & 1 & 1 & 1 & 1 & 0 & 1 & 2 & D12 \\
\hline & $9466 \mathrm{ia}$ & 2 & 1 & 2 & 2 & $1 / 2$ & 1 & 2 & 1 & 27 & 0 & 0 & 1 & 1 & 1 & 28 & 0 & 1 & 1 & 1 & 1 & 0 & 1 & 2 & D16 \\
\hline & 9467ia & 2 & 1 & 1 & 2 & $1 / 2$ & 1 & 2 & 1 & 26 & 0 & 0 & 1 & 0 & 1 & 20 & 0 & 1 & 1 & 1 & 0 & 0 & 1 & 6 & D17 \\
\hline & $9468 \mathrm{ia}$ & 2 & 1 & 1 & 2 & $1 / 2$ & 1 & 2 & 1 & 26 & 0 & 0 & 1 & 0 & 0 & 4 & 0 & 1 & 1 & 1 & 1 & 0 & 1 & 2 & D12 \\
\hline & $9469 \mathrm{ia}$ & 2 & 1 & 2 & 1 & 2 & 1 & 1 & 1 & 28 & 0 & 0 & 1 & 1 & 1 & 28 & 0 & 1 & 1 & 0 & 0 & 1 & 1 & 10 & D18 \\
\hline & $9470 \mathrm{ia}$ & 2 & 1 & 2 & 1 & 2 & 1 & 1 & 1 & 28 & 1 & 0 & 1 & 1 & 1 & 24 & 0 & 1 & 1 & 1 & 0 & 0 & 1 & 6 & D19 \\
\hline & 9471 ia & 2 & 1 & 2 & 1 & 2 & 1 & 1 & 1 & 28 & 1 & 0 & 1 & 1 & 1 & 24 & 0 & 1 & 1 & 1 & 0 & 0 & 1 & 6 & \\
\hline & $9472 \mathrm{ia}$ & 2 & 1 & 2 & 1 & 2 & 1 & 1 & 1 & 28 & 1 & 0 & 1 & 1 & 1 & 24 & 0 & 1 & 1 & 1 & 0 & 0 & 1 & 6 & \\
\hline & $9473 i a$ & 2 & 1 & 2 & 1 & 2 & 1 & 1 & 1 & 28 & 0 & 0 & 0 & 0 & 0 & 8 & 0 & 1 & 1 & 1 & 0 & 0 & 1 & 6 & D20 \\
\hline & $9474 \mathrm{ia}$ & 2 & 1 & 2 & 2 & $1 / 2$ & 1 & 2 & 1 & 27 & 0 & 0 & 1 & 1 & 1 & 28 & 0 & 1 & 1 & 0 & 0 & 1 & 1 & 10 & D21 \\
\hline & $9476 \mathrm{ia}$ & 1 & 1 & 2 & 1 & $1 / 2$ & 1 & 2 & 1 & 23 & 1 & 0 & 0 & 1 & 1 & 30 & 1 & 0 & 1 & 1 & 0 & 0 & 0 & 11 & D22 \\
\hline & 9477ia & 2 & 1 & 2 & 1 & 2 & 1 & 1 & 1 & 28 & 0 & 0 & 0 & 1 & 0 & 16 & 1 & 0 & 1 & 1 & 0 & 0 & 1 & 12 & D23 \\
\hline & $9478 \mathrm{ia}$ & 2 & 1 & 2 & 1 & 2 & 1 & 1 & 1 & 28 & 0 & 0 & 0 & 0 & 0 & 8 & 1 & 0 & 1 & 1 & 0 & 0 & 1 & 12 & D24 \\
\hline
\end{tabular}




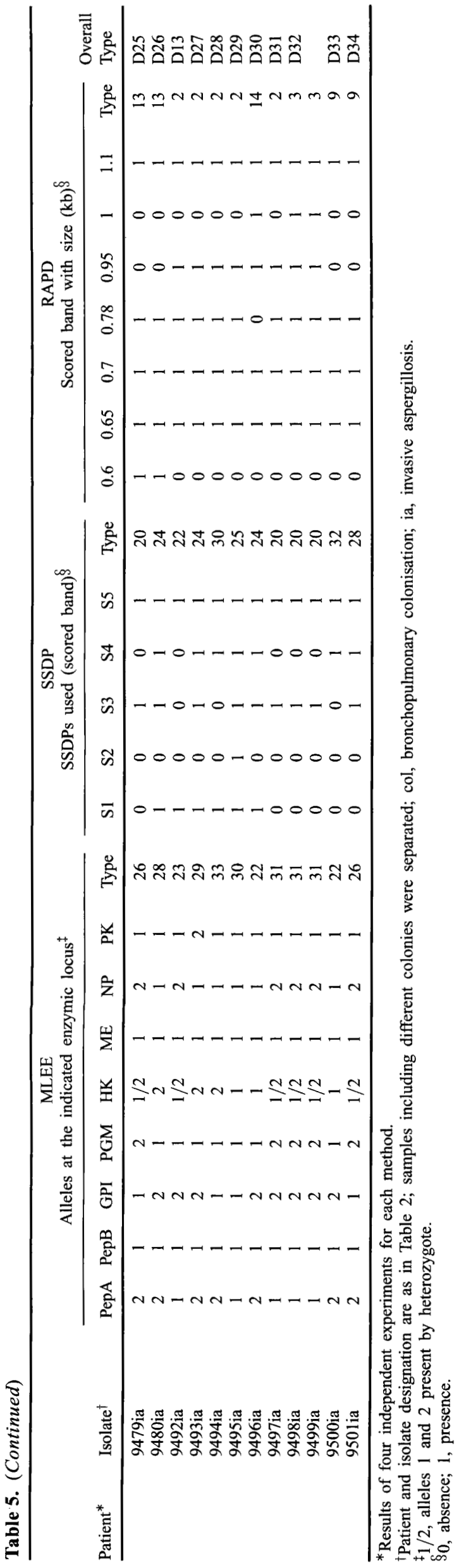

Table 6. Discriminatory power of each combination of molecular typing methods*

\begin{tabular}{lc}
\hline Typing method & Number of clusters/dendrogram \\
\hline MLEE & 7 \\
SSDP & 6 \\
RAPD & 4 \\
MLEE-SSDP & 13 \\
MLEE-RAPD & 11 \\
SSDP-RAPD & 8 \\
MLEE-SSDP-RAPD & 15 \\
\hline
\end{tabular}

*Neighbour-joining dendrograms were realised as described in Materials and methods. By dendrogram analysis, the number of distinct clusters observed was considered as a discriminatory power criterion to compare each typing method or their combination. A genetic distance of zero between two ETs implies two completely different genetic types (dissimilarity, 100\%). Genetic distance measurements give no significant percentage dissimilarity for each pair of ETs compared when $\mathrm{p}$ is $>0.05$.

greater differentiation of isolates than each molecular typing method taken separately or in pairs. This obviously contributed to a better understanding of strain relatedness in bronchial colonisation and IA in four transplant patients. The source of infection for two of the four patients was also analysed in this way. Anderson et al. demonstrated the importance of the combination of RAPD, RFLP and Southern hybridisations [10]. Lin et al. have also demonstrated that RAPD, REA and MLEE provided the best discriminatory power for typing A. fumigatus [15]. The strong polymorphism observed among the isolates in three of four patient isolates analysed in the present study was, in part, in contrast to the results obtained in other studies [19, 29, 39]. Girardin et al. [39] demonstrated that in three IA cases, isolates from two were indistinguishable with only one molecular typing method (MRS). Tang et al. [29] also demonstrated the uniqueness of IA infecting strains for two patients by RFLP and RAPD. However, they analysed only two isolates for each patient. In contrast, when a combination of several typing methods was applied to seven clinical isolates from seven neutropenic patients with evidence of IA infection, only three of the seven patients were infected by a unique strain [40]. The genetic polymorphism of isolates from aspergilloma cases have also demonstrated conflicting results in other studies [12, 21, 22, 41].

The present study demonstrated that patient $\mathrm{C}$ may have been infected by different strains. However, multiple infection in aspergilloma is more comprehensible than in IA because chronic infections are slower than IA. Therefore, these results are unexpected in relation to the rapid evolution of IA. Overall, the results presented here as well as those of other studies demonstrate the need to collect numerous isolates sequentially from each patient to analyse accurately, by different molecular typing methods, the strain relatedness and epidemiological character of aspergillosis. 
(A) Distance 0.1

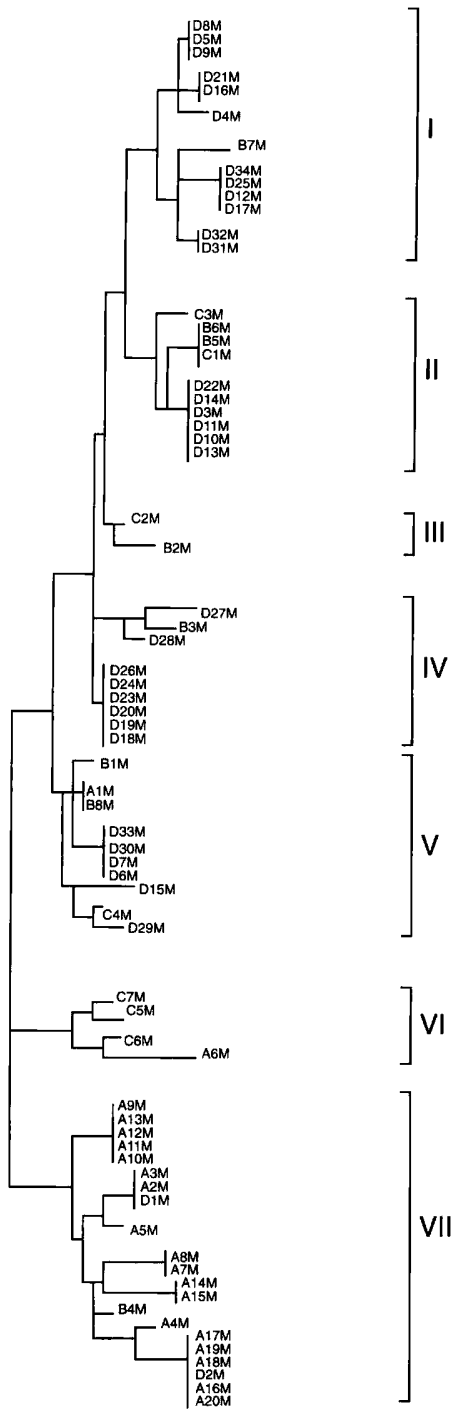

(B) Distance 0.1

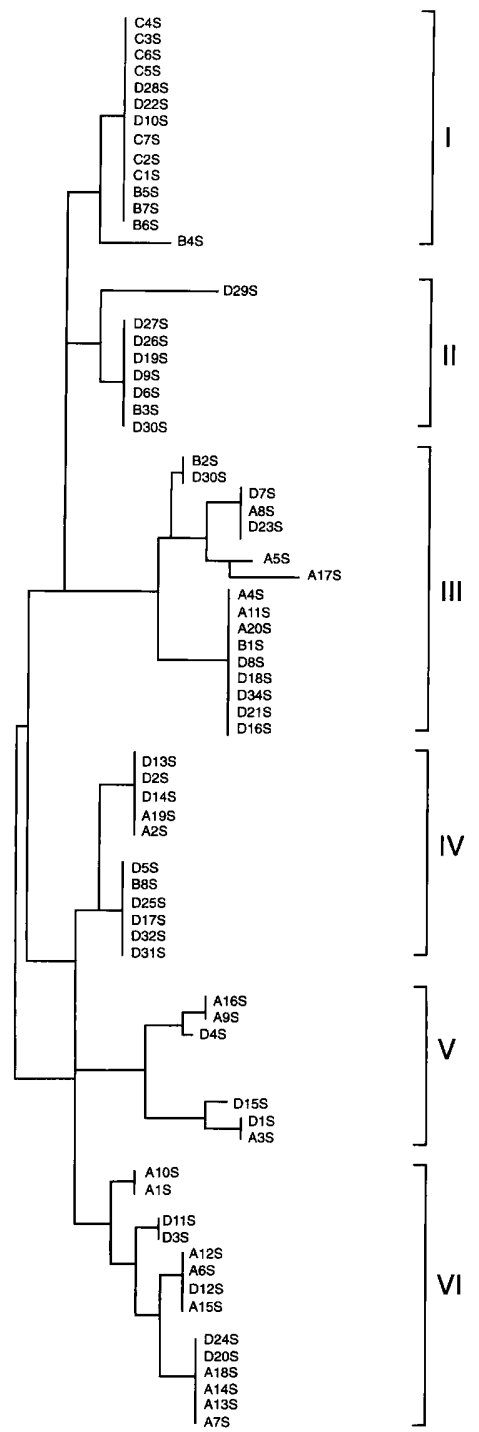

Fig. 4. (A) Genetic relationships among MLEE ETs of $A$. fumigatus. (B) Genetic relationships among SSDP ETs of $A$. fumigatus. (C) Genetic relationships among RAPD ETs of $A$. fumigatus. (D) Genetic relationships among overall ETs of $A$. fumigatus when MLEE-SSDP-RAPD were combined. The dendrograms were created by the neighbour-joining method (see Materials and methods). ET designations are as in Tables 1 and 2. Subpopulations are designated by Roman numerals. A genetic distance of zero between two ETs implies two completely different genetic types (dissimilarity, $100 \%$ ). Genetic distance measurements give no significant percentage dissimilarity for each pair of ETs compared when $\mathrm{p}$ is $>0.05$.

Several explanations can account for the occurrence of a multiple aspergillosis infection. First, it is possible that particular strains were brought to the hospital by the infected patient. Alternatively, it is possible that the patient may have been infected by conidia derived from an environmental source within or in the proximity of the hospital. It was observed that strains XII-XIV appeared to be specific to the environment of patient A (Grenoble, France), strains V and VI appeared to be specific to patient $C$ (Milan, Italy), and strains IV and $\mathrm{X}$ to be specific to patient $\mathrm{D}$ (Lyon, France). However, no construction activities or building demolition took place in, or in the immediate vicinity of, the hospital during the period of these episodes of aspergillosis. Thus, it may be the result of generally elevated numbers of Aspergillus spores present in the outside environment in cyclical periods $[42,43]$. If strains I-III, VII and VIII were isolated from several patients we would say that there is no geographical specialisation of the A. fumigatus strains. Extensive genotypic biodiversity for $A$. fumigatus could be the result of such predominance operating over time. However, the occurrence of multiple infections may not be surprising in regard to the aerial propagation of this fungus and by $A$. fumigatus genomic dynamics [9].

These results point out the necessity of combining different molecular typing methods to reveal the true source of infection. As the three methods demon- 
(C)

Distance 0.1

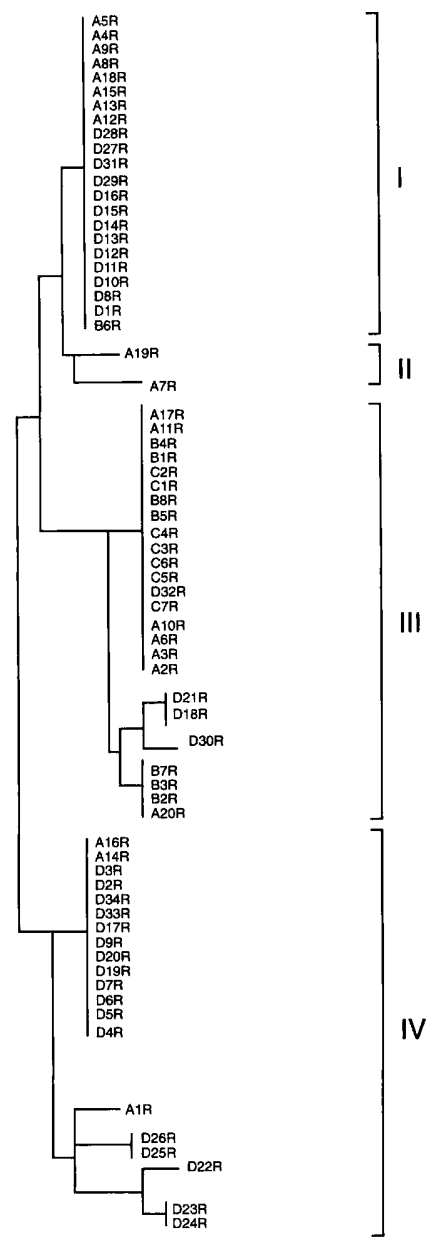

III
(D) Distance 0.1

Fig. 4. (Continued)

strated concordance in the study of patient B isolates, this suggests that the infection was nosocomially acquired in this case. Both Girardin et al. [39] and Paugam et al. [19] have also suggested a case of nosocomially acquired infection. However, the results of the present study, as well as those of others, underline the need to isolate numerous strains from the patient and the environment in the early stages of aspergillosis. In contrast, complete concordance among the three molecular typing methods was not seen in the analysis of isolates from patients $A$ and $C$. With typing by MLEE and RAPD, the origin of infection in

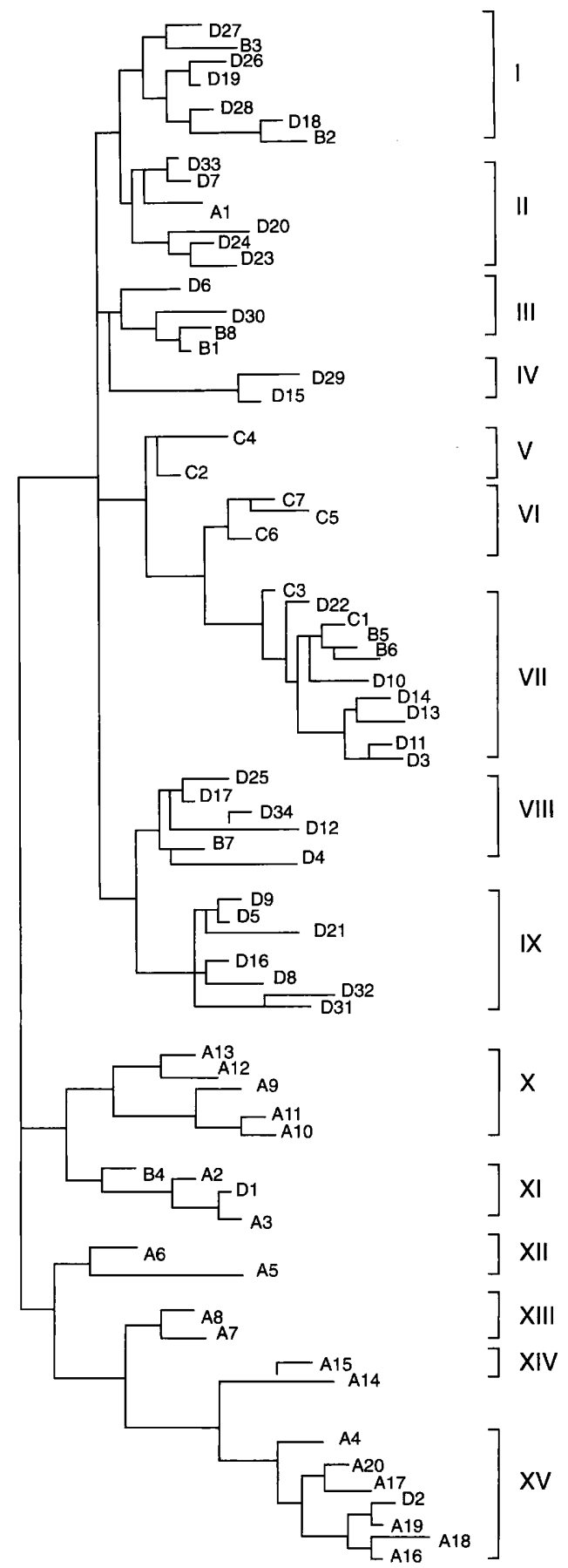

patient A was still unknown. By SSDP only, it could be concluded that type A10 may be the cause of infection. When isolates from patient $\mathrm{C}$ were analysed, only MLEE demonstrated polymorphism of the samples. MLEE can be considered more discriminating than the other two techniques because it uses 12 markers, and this was necessary to reveal the diversity of infective strains. The reasons why MLEE revealed such high polymorphism may have an obvious explanation. First, one difference between two MLEE types could be the result of a genetic event such as nucleic acid mutation, deletion, or insertion. Second, 
the lack of expression of some alleles by genetic repression as well as allelic degradation or modification after post-traductional processes could modify the global protein electrophoretic charge. While good agreement between methods was generally observed, Lin et al. [15] have also shown that the concordance among methods was not always obvious. Rinyu et al. [20] also demonstrated the lack of strict correlation between iso-enzyme and RAPD data. Many strains that were found to be closely related by iso-enzyme analysis gave different RAPD patterns. In contrast, Tang et al. demonstrated a good correlation between RFLP and RAPD patterns in a study of a limited number of isolates [29]. The results of the present study demonstrate differences in sensitivity of the MLEE, SSDP and RAPD typing methods after statistical analysis. This has also been observed with a combination of typing methods for Candida albicans [25] and Cryptococcus neoformans [27]. It may be caused by discrepancies in qualitative and quantitative polymorphic markers between the methods used at different levels, genomic and proteic. Taken together, these results and those from other studies underline the fact that when multiple methods are applied, the discriminatory power increases whereas concordance among methods decreases.

In conclusion, these results demonstrate the importance of using a combination of various typing methods each applied independently in different laboratories. They must be applied with the understanding that more than one approach may be necessary to achieve optimal strain discrimination. In the next EBGA network epidemiological studies, results from the three molecular typing methods MLEE (Montpellier, France), RAPD (Brussels, Belgium) and SSDP (Grenoble, France) will be associated with results from other typing methods developed in Lyon (France) and Leeds (UK). The involvement of numerous techniques may allow the genetic distance between ETs to be measured more accurately. In this way, a better analysis of genotypic relatedness could contribute to a more precise definition of infective strains and a full understanding of the epidemiology of A. fumigatus.

This work was supported in part by a research grant from the European Union, contract BMH4-97-2481 (CEE-BIOMED2). SSDP analysis was supported by funds from the 'Recherche Clinique-CHU Grenoble', France. P. M. was supported by a grant from the Agence de l'environnement et de la Maîtrise de l'Énergie (ADEME), Paris, France. We gratefully acknowledge the technical assistance of E. Coursange and S. Arnavielhe.

\section{References}

1. Rogers TR. Nosocomial infections associated with bone marrow and other transplants. Curr Opin Infect Dis 1994; 7: 484-487.

2. Saral R. Candida and Aspergillus infections in immunocompromised patients: an overview. Rev Infect Dis 1991; 13: $487-492$
3. Denning DW. Invasive aspergillosis in immunocompromised patients. Curr Opin Infect Dis 1994; 7: 456-462.

4. Pla MP, Berenger J, Arzuaga JA, Banares R, Polo JR, Bouza E. Surgical wound infection by Aspergillus fumigatus in liver transplant recipients. Diagn Microbiol Infect Dis 1992; 15: $703-706$.

5. Yeldandi V, Laghi F, McCabe MA et al. Aspergillus and lung transplantation. J Heart Lung Transplant 1995; 14: 883-890.

6. Wald A, Leisenring W, van Burik JA, Bowden RA. Epidemiology of Aspergillus infections in a large cohort of patients undergoing bone marrow transplantation. J Infect Dis 1997; 175: $1459-1466$.

7. Loudon KW, Coke AP, Burnie JP, Lucas GS, Liu Yin JA. Invasive aspergillosis: clusters and sources? $J$ Med Vet Mycol 1994; 32: 217-224.

8. Denning DW, Clemons KV, Hanson LH, Stevens DA. Restriction endonuclease analysis of total cellular DNA of Aspergillus fumigatus isolates of geographically and epidemiologically diverse origin. $J$ Infect Dis 1990; 162: 1151-1158.

9. Rodriguez E, De Meeüs T, Mallié M et al. Multicentric epidemiological study of Aspergillus fumigatus isolates by multilocus enzyme electrophoresis. J Clin Microbiol 1996; 34: 2559-2568.

10. Anderson MJ, Gull K, Denning DW. Molecular typing by random amplification of polymorphic DNA and M13 Southern hybridization of related paired isolates of Aspergillus fumigatus. J Clin Microbiol 1996; 34: 87-93.

11. Aufauvre-Brown A, Cohen J, Holden DW. Use of randomly amplified polymorphic DNA markers to distinguish isolates of Aspergillus fumigatus. $J$ Clin Microbiol 1992; 30: 2991-2993.

12. Denning DW, Shankland GS, Stevens DA. DNA fingerprinting of Aspergillus fumigatus isolates from patients with aspergilloma. J Med Vet Mycol 1991; 29: 339-342.

13. Girardin H, Latgé JP, Srikantha T, Morrow B, Soll DR. Development of DNA probes for fingerprinting Aspergillus fumigatus. J Clin Microbiol 1993; 31: 1547-1554.

14. Leenders A, Van Belkum A, Janssen S et al. Molecular epidemiology of apparent outbreak of invasive aspergillosis in a hematology ward. J Clin Microbiol 1996; 34: 345-351.

15. Lin D, Lehmann PF, Hamory $\mathrm{BH}$ et al. Comparison of three typing methods for clinical and environment isolates of Aspergillus fumigatus. J Clin Microbiol 1995; 33: 1596-1601.

16. Mondon P, Thélu J, Lebeau B, Ambroise-Thomas P, Grillot R. Virulence of Aspergillus fumigatus strains investigated by random amplified polymorphic DNA analysis. $J \mathrm{Med}$ Microbiol 1995; 42: 299-303.

17. Mondon P, De Champs C, Donadille A, Ambroise-Thomas P, Grillot R. Variation in virulence of Aspergillus fumigatus strains in a murine model of invasive pulmonary aspergillosis. $J$ Med Microbiol 1996; 45: 186-191.

18. Mondon P, Brenier MP, Coursange E, Lebeau B, Ambroise Thomas P, Grillot R. Molecular typing of Aspergillus fumigatus strains by sequence-specific DNA primer (SSDP) analysis. FEMS Immunol Med Microbiol 1997; 17: 95-102.

19. Paugam A, Bougnoux ME, Robert $\mathrm{F}$ et al. Use of randomly amplified polymorphic DNA markers (RAPD) to demonstrate nosocomial contamination in a case of lethal invasive aspergillosis. $J$ Hosp Infect 1995; 29: 158-161.

20. Rinyu E, Varga J, Ferenczy M. Phenotypic and genotypic analysis of variability in Aspergillus fumigatus. $J$ Clin Microbiol 1995; 33: 2567-2575.

21. Burnie JP, Coke A, Matthews RC. Restriction endonuclease analysis of Aspergillus fumigatus DNA. J Clin Pathol 1992; 45: $324-327$

22. Girardin H, Sarfati J, Kobayashi H, Bouchara JP, Latgé JP. Use of DNA moderately repetitive sequence to type Aspergillus fumigatus isolates from aspergilloma patients. $J$ Infect Dis 1994; 169: 683-685.

23. Burnie JP, Matthews RC, Clark I, Milne LJR. Immunoblot fingerprinting Aspergillus fumigatus. J Immunol Methods 1989; 118: $179-186$.

24. Symoens F, Viviani MA, Nolard N. Typing by immunoblot of Aspergillus fumigatus from nosocomial infections. Mycoses 1992; 36: 229-237.

25. Matsuda $\mathrm{H}$, Kohno $\mathrm{S}$, Maesaki $\mathrm{S}$ et al. Application of ubiquinone systems and electrophoretic comparison of enzymes to identification of clinical isolates of Aspergillus fumigatus and several other species of Aspergillus. J Clin Microbiol 1992; 30: 1999-2005. 
26. Nealson KH, Garber ED. An electrophoretic survey of esterases, phosphatases, and leucine aminopeptidases in mycelial extracts of species of Aspergillus. Mycologia 1967; 59: 330-336.

27. Boerlin P, Boerlin-Petzold F, Goudet $\mathrm{J}$ et al. Typing Candida albicans oral isolates from human immunodeficiency virusinfecting patients by multilocus enzyme electrophoresis and DNA fingerprinting. J Clin Microbiol 1996; 34: 1235-1248.

28. Brandt ME, Hutwagner LC, Kuykendall J, Pinner RW, and the Cryptococcal Disease Active Surveillance Group. Comparison of multilocus enzyme electrophoresis and random amplified polymorphic DNA analysis for molecular subtyping of Cryptococcus neoformans. J Clin Microbiol 1995; 33: 1890-1895.

29. Tang CM, Cohen J, Rees AJ, Holden DW. Molecula epidemiological study of invasive pulmonary aspergillosis in renal transplantation unit. Eur $J$ Clin Microbiol Infect Dis 1994; 13: 318-321.

30. Raper KB, Fennel DI. The genus Aspergillus. Baltimore, Williams and Wilkins. 1965.

31. Harris H, Hopkinson J. Handbook of enzyme electrophoresis in human genetics. Amsterdam, Elsevier/North-Holland Publishing. 1976.

32. Pasteur N, Pasteur G, Bonhomme F, Catalan J, Britton-Davidian J. Manuel technique de génétique par électrophorèse des proteines. Techniques et Documentations. Paris, Lavoisier. 1987.

33. Richardson BJ, Baverstock PR, Adams M. Allozyme electrophoresis: a handbook for animal systematics and population studies. Sydney, Academic Press Australia. 1986.

34. Van Vaerenbergh B, Grootaert B, Moens W. Validation of a method for the preparation of fungal genomic DNA for polymerase chain reaction (PCR) and random amplification of polymorphic DNA (RAPD). J Mycol Med 1995; 3: 133-139.

35. White T, Bruns T, Lee S, Taylor J. Analysis of phylogenetic relationships by amplification and direct sequencing of ribosomal RNA genes. In: Innis M, Gelfand D, Sninsky J, White $\mathrm{T}$ (eds) PCR protocols: a guide to methods and applications. Orlando, FL, Academic Press. 1990: 315-322.

36. Saitou $N$, Nei $M$. The neighbor-joining method: a new method for reconstructuring phylogenetic trees. Mol Biol Evol 1987; 4: 406-425.

37. Hillis DM, Huelsenbeck JP, Cunningham CW. Application and accuracy of molecular phylogenics. Science 1994; 264: $671-677$.

38. Lee W, Burnie J, Matthews R. Fingerprinting Candida albicans. J Immunol Methods 1986; 93: 177-182.

39. Girardin H, Sarfati J, Traoré F, Dupuy Camet J, Deroin F, Latgé JP. Molecular epidemiology of nosocomial invasive aspergillosis. J Clin Microbiol 1994; 32: 684-690.

40. Loudon KW, Burnie JP, Coke AP, Matthews RC. Application of polymerase chain reaction to fingerprinting Aspergillus fumigatus by random amplification of polymorphic DNA. $J$ Clin Microbiol 1993; 31: 1117-1121.

41. Rath PM, Ratjen F, Ansorg R. Genetic diversity among isolates of Aspergillus fumigatus in patients with cystic fibrosis. Zentralbl Bakteriol 1997; 285: 450-455.

42. Beyer J, Schwartz S, Heinemann V, Siegert W. Strategies in prevention of invasive pulmonary aspergillosis in immunosuppressed or neutropenic patients. Antimicrob Agents Chemother 1994; 38: 911-917.

43. Rhame FS. Prevention of nosocomial aspergillosis. J Hosp Infect 1991; 18 Suppl A: 466-472. 